\title{
Physicochemical and Pharmacokinetic Characterization of Amorphous Solid Dispersion of Meloxicam with Enhanced Dissolution Property and Storage Stability
}

\author{
Masanori Ochi, ${ }^{1,2}$ Keisuke Kimura, ${ }^{1}$ Atsushi Kanda, ${ }^{1}$ Takaki Kawachi, ${ }^{1}$ Akitoshi Matsuda, ${ }^{1}$ \\ Kayo Yuminoki, ${ }^{1}$ and Naofumi Hashimoto ${ }^{1}$
}

Received 31 May 2015; accepted 24 September 2015; published online 5 October 2015

\begin{abstract}
The aim of the present study was to develop amorphous solid dispersion (ASD) of meloxicam (MEL) for providing rapid onset of action. ASDs of MEL with polyvinylpyrrolidone (PVP) K-30 (MEL/ PVP), HPC-SSL (MEL/HPC), and Eudragit EPO (MEL/EPO) were prepared. The physicochemical properties were characterized by focusing on morphology, crystallinity, dissolution properties, stability, and the interaction of MEL with coexisting polymers. MEL/EPO was physicochemically stable after storage at $40^{\circ} \mathrm{C} / 75 \% \mathrm{RH}$ for 30 days. In contrast, recrystallization of MEL was observed in MEL/PVP and MEL/HPC at $40^{\circ} \mathrm{C} / 50 \% \mathrm{RH}$ for 30 days. Infrared spectroscopic studies and ${ }^{1} \mathrm{H}$ NMR analyses of $\mathrm{MEL} / \mathrm{EPO}$ revealed that Eudragit EPO interacted with MEL and reduced intermolecular binding between MEL molecules. Intermolecular interaction of drug molecules is necessary for the formation of crystalline. Thus, the interaction of MEL with Eudragit EPO and interruption of the formation of supramolecular interaction between MEL molecules might lead to the inhibition of crystal growth of MEL. Of all the MEL solid dispersions prepared, MEL/EPO showed the largest improvement in dissolution behavior. Oral administration of MEL/EPO to rats showed rapid and enhanced MEL exposure with a 2.4-fold increase in bioavailability compared with crystalline MEL. Based on these findings, $\mathrm{MEL} / \mathrm{EPO}$ was physicochemically stable and provided a rapid onset of action and enhanced bioavailability after oral administration.
\end{abstract}

KEY WORDS: amorphous; dissolution; FTIR; interaction; stability.

\section{INTRODUCTION}

Meloxicam (MEL) is among the most potent nonsteroidal anti-inflammatory drugs (NSAIDs). MEL has been used for treatment of rheumatoid arthritis (1), osteoarthritis (2), and postoperative pain (3). However, the rapid onset of pharmaceutical effects of MEL is limited because of the slow oral absorption. MEL has a low molecular weight (351.4) and $\mathrm{pKa}$ values of 1.1 (hydroxyl group) and 4.2 (thiazole group). MEL was found to be poorly soluble in water, and the solubility in solution at $\mathrm{pH} 1.2$ or 4.0 was reported to be as low as $c a .0 .6 \mu \mathrm{g} / \mathrm{mL}$ (4). The limited solubility in acidic solution is thought to cause the slow absorption after oral administration. Solving solubility problems is a major challenge for the pharmaceutical industry in the development of new drug candidates, with approximately $70 \%$ of new drug candidates having been identified as either insoluble or poorly soluble in water (5).

The amorphous solid dispersion (ASD) technique is one of the most effective and simple methods for improving the

\footnotetext{
$\overline{{ }^{1} \text { Department of Pharmaceutical Physicochemistry, Faculty of }}$ Pharmaceutical Sciences, Setsunan University, 45-1 Nagaotoge-cho, Hirakata, Osaka 573-0101, Japan.

${ }^{2}$ To whom correspondence should be addressed. (e-mail: 12d403om@edu.setsunan.ac.jp)
}

dissolution behavior and bioavailability of poorly soluble drugs (6). However, a major issue for ASD is the crystallization of drugs during storage (7). The excess free energy of an amorphous solid drives nucleation and growth of the crystal of the drug molecules (8). Crystallization of an amorphous drug could lead to a reduction in dissolution rate, causing a reduction of bioavailability. Therefore, stabilization of the ASD is essential for ensuring sufficient therapeutic efficacy.

Although some ASD formulations of MEL were reported $(9,10)$, drug-polymer amorphous solid dispersions of MEL with storage stability have never been reported yet. Thus, this study aimed to develop a physicochemically stable MEL ASD with improved dissolution behavior in order to shorten the onset of pharmacological effects. The physicochemical properties were characterized by polarized light microscopy, X-ray powder diffraction (XRPD) analyses before and after storage, and dissolution test in acidic media. It has been reported that ASDs can be physicochemically stabilized by drug-polymer interaction $(11,12)$. Intermolecular interaction of drug molecules is necessary for the crystal nucleation and growth. A drug-polymer interaction can reduce the formation rate of intermolecular interaction between drug molecules, so the rate of crystal nucleation and growth was reduced. Thus, the interaction of MEL with Eudragit EPO and interruption of the formation of supramolecular interaction between MEL 
molecules might lead to the inhibition of crystal growth of MEL. The molecular interaction between MEL and hydrophilic polymer in ASD was assessed by Fourier transform infrared (FTIR) spectral analysis for identification of drugpolymer interaction affecting the stabilization of ASD. The physicochemical stability and dissolution profile suggested the most reliable ASD of MEL for in vivo testing. The pharmacokinetic profile of MEL was evaluated after oral administration of the ASD in rats.

\section{MATERIALS AND METHODS}

\section{Materials}

Polyvinylpyrrolidone (PVP, Fig. 1a) and 1,4-dioxane were purchased from Wako Pure Chemical Industries, Ltd. (Osaka, Japan). Hydroxypropyl cellulose (HPC, Fig. 1b) was obtained from Nippon Soda Co., Ltd. (Tokyo, Japan). Eudragit EPO ((poly(butylmethacrylate-co-(2-dimethylaminoethyl)methacrylateco-methyl methacrylate) 1:2:1, Fig. 1c) was provided by Evonik Industries (Darmstadt, Germany). Acetonitrile (liquid chromatography grade) was purchased from Kanto Chemical (Tokyo, Japan). Crystalline MEL (Fig. 1d) and Mobic (marketed formulation of MEL) were purchased from Tokyo Chemical Industry Co., Ltd. (Tokyo, Japan) and Boehringer Ingelheim Co., Ltd., (Kobe, Japan), respectively. All other chemicals were purchased from commercial sources.

\section{Solid Dispersion Preparation}

Crystalline MEL (30 mg) and hydrophilic polymer (90 mg) were dissolved in $30 \mathrm{~mL}$ of 1,4-dioxane solution. The solution was mixed well, frozen in liquid nitrogen, and freeze-dried using a FD-81 freeze dryer (Tokyo Rikakikai,
Tokyo, Japan). ASD containing 25\% (w/w) MEL was obtained.

\section{HPLC/UV Analysis}

The concentration of MEL was determined by an absolute calibration method using a high-performance liquid chromatography (HPLC) system equipped with a diode array detector (Shimadzu, Kyoto, Japan). The HPLC system consisted of an LC-20A solvent delivery unit with highpressure flow-line selection valves, an SIL-20A auto sampler, a CTO-20A column oven, and an SPD-M20A diode array detector connected to the LC solution software. Chromatography was conducted using a COSMOSIL $5 \mathrm{C}_{18}$-AR-II column (particle size $5 \mu \mathrm{m}$, column dimensions $4.6 \mathrm{~mm} \times 150 \mathrm{~mm}$ ). Column temperature was maintained at $40^{\circ} \mathrm{C}$, and the samples were separated using a mobile phase consisting of $20 \mathrm{mM}$ phosphate buffer $(\mathrm{pH} 7.0)$ and acetonitrile $(3: 7, v / v)$ at a flow rate of $0.5 \mathrm{~mL} / \mathrm{min}$. The diode array detector was set at $360 \mathrm{~nm}$.

\section{XRPD Analysis}

XRPD patterns were collected with an RINT diffractometer (Rigaku Co., Tokyo, Japan) with $\mathrm{Cu} \mathrm{K} \alpha$ radiation generated at $40 \mathrm{~mA}$ and $40 \mathrm{kV}$. Data were obtained at angles from $5^{\circ}$ to $40^{\circ}(2 \theta)$ at a step size of $0.2^{\circ}$ and a scanning speed of $5^{\circ}$ $\min$.

\section{Polarized Light Microscopy}

Representative polarized light microscopy (PLM) images of MEL samples were taken using a VHX-5000 microscope (Keyence, Osaka, Japan). MEL samples were examined<smiles>CC(C)(C)C(N1CCCC1=O)C(C)(C)C</smiles>

(C)<smiles>CCCCCCOC(=O)C(C)(CC(C)(C)C(=O)OC)CC(C)(C)C(=O)OCCN(C)C</smiles>

(B)

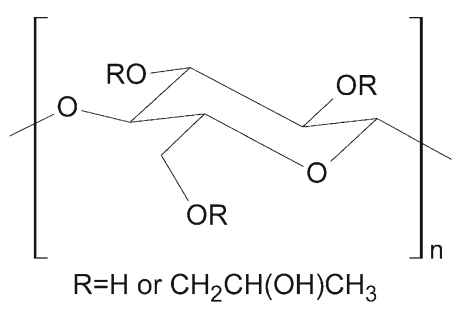

(D)<smiles>Cc1cnc(NC(=O)C2=C(O)c3ccccc3S(=O)(=O)N2C)s1</smiles>

Fig. 1. Chemical structures of a PVP K-30, b HPC-SSL, c Eudragit EPO, and d meloxicam (MEL) 
under various conditions, including differential interference contrast, using slightly uncrossed polarizers.

\section{Stability Testing}

Stability studies of the MEL samples were carried out at $40 \pm 2{ }^{\circ} \mathrm{C} / 75 \% \mathrm{RH}$ or $40 \pm 2{ }^{\circ} \mathrm{C} / 50 \% \mathrm{RH}$ for 30 days in a stability chamber. MEL samples were stored in open bottles. Relative humidity of $75 \%$ or $50 \%$ was maintained by the inclusion in the chamber of a suspension of sodium chloride or magnesium nitrate, respectively. Following storage, the samples were subjected to the XRPD and PLM experiments.

\section{Fourier Transform Infrared Spectroscopy}

FTIR analysis was conducted to evaluate drug-polymer interactions. Powder samples were prepared by mixing approximately 2 to $3 \mathrm{mg}$ of MEL and the physical mixture or ASD with $300 \mathrm{mg}$ of $\mathrm{KBr}$, and the mixture was pressed to prepare a $\mathrm{KBr}$ disk. Spectra were recorded with IR solution software (Shimadzu, Kyoto, Japan), and 40 scans were performed with a resolution of $4 \mathrm{~cm}^{-1}$.

\section{Nuclear Magnetic Resonance Analysis}

Hydrogen-1 Nuclear Magnetic Resonance Analysis $\left({ }^{1} \mathrm{H}\right.$ NMR) spectra of MEL samples with or without Eudragit EPO were recorded on a JEOL ECA 500 spectrometer (JEOL Ltd., Tokyo, Japan) using dimethylsulfoxide- $d_{6}(99.9 \%$ D, Wako Pure Chemical Industries, Ltd.) as a solvent. ${ }^{1} \mathrm{H}$ NMR spectra of MEL (6 mg) with or without Eudragit EPO (6 mg) were recorded at a sample temperature of $293 \mathrm{~K}$.

\section{Dissolution Test}

Dissolution tests were carried out in $900 \mathrm{~mL}$ of $\mathrm{HCl}$ solution ( $\mathrm{pH}$ 1.2) using Japanese Pharmacopeia dissolution apparatus I (NTR-VS6, Toyama Sangyo Co., Ltd., Osaka, Japan). The rotating basket method was used, with constant stirring at $100 \mathrm{rpm}$ and incubation at $37^{\circ} \mathrm{C}$. Each MEL sample was weighed to keep the total amount of MEL in the dissolution vessel constant at $15 \mathrm{mg}$, corresponding to the clinical maximum dose. The condition of dissolution tests were nonsink condition. Aliquots ( $1 \mathrm{~mL}$ each) of sample were collected at $15,30,60,90$, and $120 \mathrm{~min}$ using a pipette; the removed volume was not replaced. The sampling site was $1 \mathrm{~cm}$ from the test vessel wall at $c a .25 \mathrm{~mm}$ below the surface of the medium. The samples were centrifuged at $15,000 \times \mathrm{g}$ for $15 \mathrm{~min}$ to remove insoluble materials. The concentration of MEL in the supernatant was determined by HPLC/UV analysis as described above. Equivalence of dissolution profiles of MEL/EPO before and after storage at $40^{\circ} \mathrm{C} / 75 \% \mathrm{RH}$ for 30 days was confirmed using the similarity factor $(f 2)$. The similarity factor is a logarithmic transformation of the sumsquared error of differences between the test and reference product over all time points.

$$
f 2=50 \times \log \left[\left\{\left(1+\sum(R t-T t) \times 1 / N\right)\right\}^{-0.5}\right]
$$

Rt and $\mathrm{Tt}$ are the individual or mean percentage dissolved at each time point, $t$, for the reference and test dissolution profiles, respectively. $N$ is the number of time points. $f 2$ represents the closeness of two comparative formulations. Generally, similarity factor in the range of $50-100$ is acceptable according to US FDA.

\section{Animals}

Male Sprague-Dawley (SD) rats (290.9-407.4 g in weight; Japan SLC, Shizuoka, Japan) were housed in the laboratory with free access to food and water. All procedures used in the present study were approved by the Ethical Review Committee of Setsunan University.

\section{Plasma Concentration of MEL after Oral Administration}

Male SD rats were fasted overnight before the experiment but given free access to water. A dose equivalent to $1.0 \mathrm{mg}-\mathrm{MEL} / \mathrm{kg}$ body weight was administered orally. The suspensions were prepared with distilled water; the dosing concentration was $0.5 \mathrm{mg}-\mathrm{MEL} / \mathrm{mL}$. Blood samples were

(A)

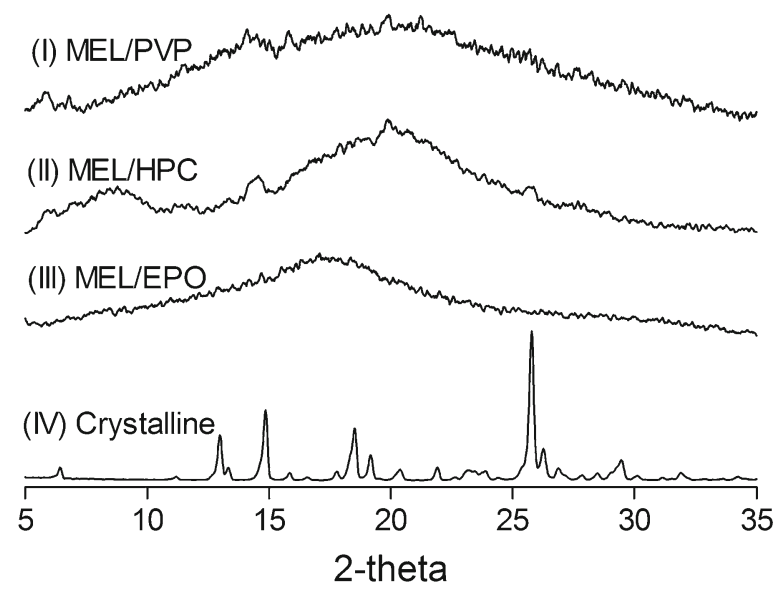

(B)

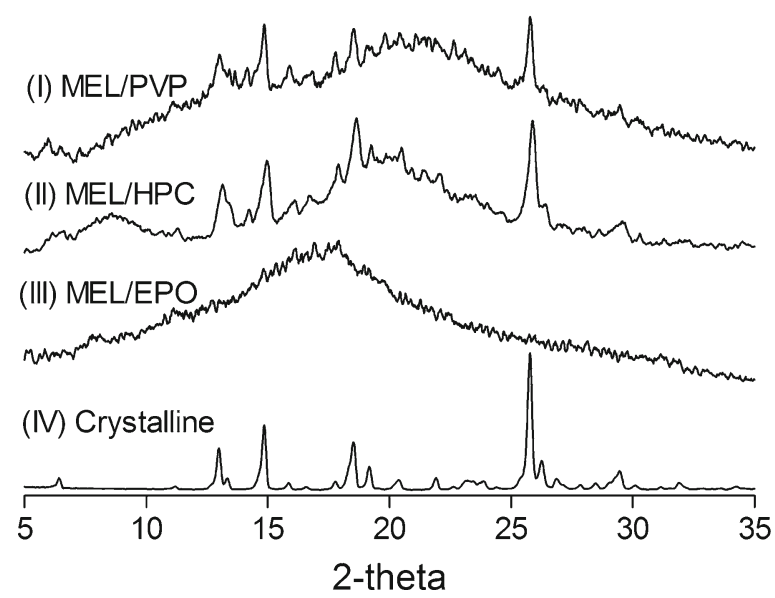

Fig. 2. Physicochemical characterization of MEL formulation using $\mathrm{X}$-ray powder diffraction a soon after preparation and $\mathbf{b}$ after storage at $40^{\circ} \mathrm{C} / 75 \% \mathrm{RH}$ or $40^{\circ} \mathrm{C} / 50 \% \mathrm{RH}$ for 30 days. (I) MEL/PVP, (II) MEL/HPC, (III) MEL/EPO, and (IV) crystalline MEL 
collected from the jugular vein at $0,0.5,1,2,4$, and $8 \mathrm{~h}$ after oral administration. Each blood sample was centrifuged at $1000 \times g$ for $10 \mathrm{~min}$ to prepare plasma samples. Plasma MEL concentration was determined by HPLC/UV. Briefly, $75 \mu \mathrm{L}$ of acetonitrile was added to $75 \mu \mathrm{L}$ of plasma sample and then centrifuged at $10,000 \times g$ for $5 \mathrm{~min}$. The supernatant was analyzed on Shimadzu HPLC system using a gradient mobile phase consisting of (A) $20 \mathrm{mM}$ phosphate buffer ( $\mathrm{pH} 7.0$ ) and $(\mathrm{B})$ acetonitrile $(3 / 7, v / v)$. The gradient condition of the mobile phase was $0-0.5 \mathrm{~min}, 70 \% \mathrm{~A}$; $0.5-5.5 \mathrm{~min}, 70 \%-55 \%$ A; 5.51-6.0 $\mathrm{min}, 40 \%$ A. The flow rate was $0.5 \mathrm{~mL} / \mathrm{min}$. $C_{\max }$ and the area under the curve of plasma MEL concentration were calculated using GastroPlus version 8.4 (Simulations Plus Inc., Lancaster, CA, USA).

\section{Statistical Analysis}

Comparisons between the two experimental groups were carried out using the $F$-test and unpaired Students $t$-test. All statistical tests were two-sided, and A $P$ value of less than 0.05 was considered statistically significant for all analyses.

\section{RESULTS}

\section{XRPD Analysis and PLM Observations}

In the present study, ASD formulations of MEL were freeze-dried with PVP K-30 (MEL/PVP), HPC-SSL (MEL/ HPC), or Eudragit EPO (MEL/EPO). The physical states of MEL in the prepared ASDs were evaluated by XRPD analyses. As shown in Fig. 2a, several intense peaks were observed in the XRPD pattern of crystalline MEL; these peaks indicated the anhydrous form of MEL (Form I) (13). In contrast, all of the prepared ASDs of MEL showed a halo diffraction pattern, and typical peaks of form I or other known crystalline forms were negligible (14). PLM experimental analyses were performed for further characterization of ASD formulations of MEL because the sensitivity of PLM analysis for detection of crystallinity was much higher than that of XRPD analysis (Fig. 3). Although MEL in the ASD formulations was expected to be amorphous, the PLM observation revealed that MEL recrystallized slightly in MEL/HPC and MEL/PVP as evidenced by weak birefringence (Fig. 3-I, II). Only MEL/EPO showed no birefringence.

\section{Stability Testing}

Since stability of the ASD is indispensable for sufficient therapeutic efficacy, a solid-state stability study was conducted for these ASD formulations of MEL. Following storage at $40^{\circ} \mathrm{C} / 75 \% \mathrm{RH}$ or $40^{\circ} \mathrm{C} / 50 \% \mathrm{RH}$ for 30 days, the ASD formulations were subjected to XRPD analyses to evaluate transition to a crystalline state. MEL/EPO maintained a halo pattern after 30 days storage at $40^{\circ} \mathrm{C} / 75 \% \mathrm{RH}$ (Fig. 2b-III). However, MEL/PVP and MEL/HPC were converted to semisolid materials (data not shown) under the same conditions; therefore XRPD analyses of MEL/PVP and MEL/HPC formulations were impossible. Thus, storage stability testing was conducted at $40^{\circ} \mathrm{C} / 50 \% \mathrm{RH}$ for comparison of solid-state stability of ASDs. None of the ASD formulations became semisolid after 30 days storage at $40^{\circ} \mathrm{C} / 50 \% \mathrm{RH}$. MEL/EPO maintained a halo pattern after 30 days storage at $40^{\circ} \mathrm{C} / 50 \%$ $\mathrm{RH}$, while several intense peaks were observed by XRPD analysis of MEL/PVP and MEL/HPC following storage under
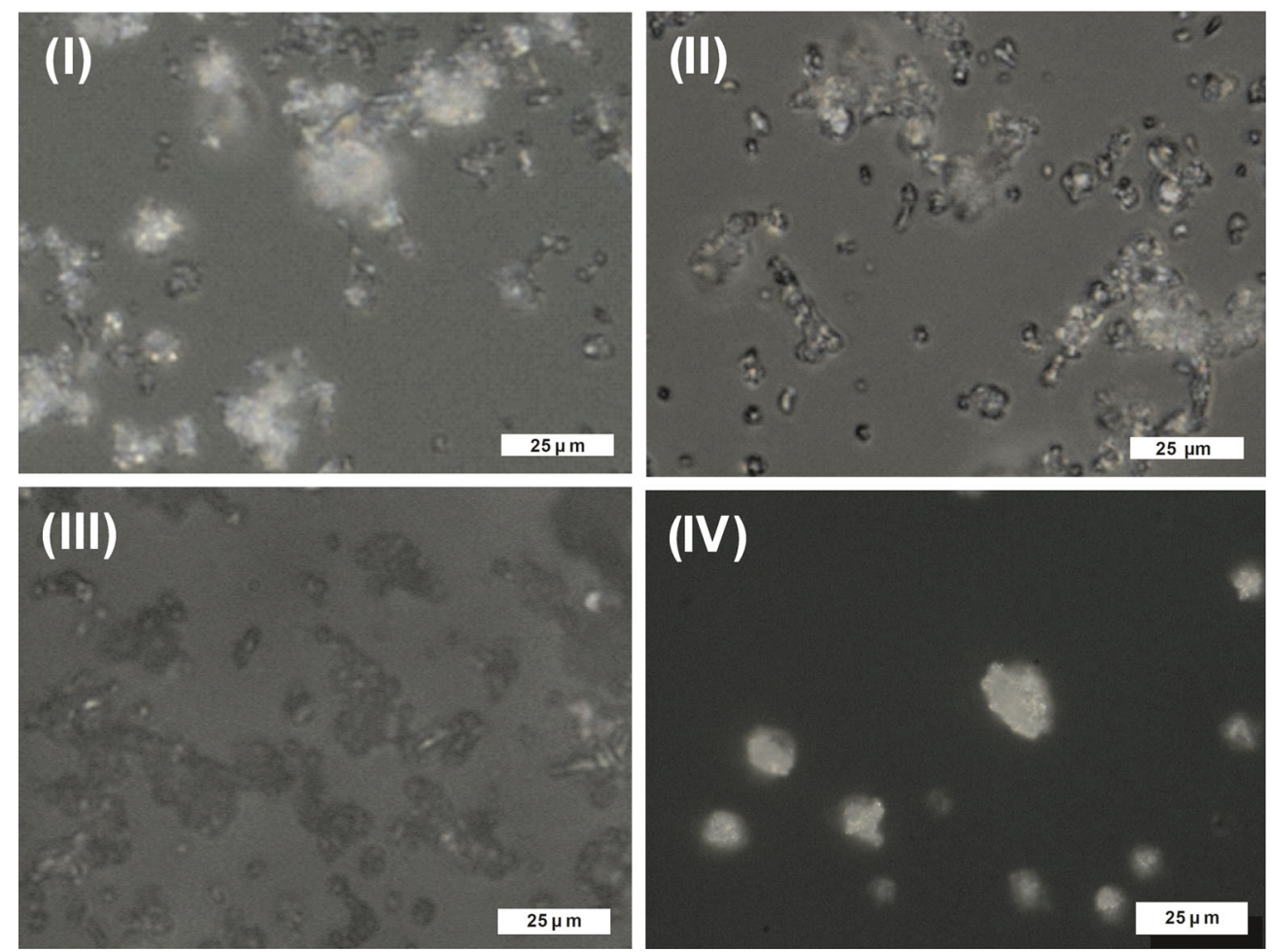

Fig. 3. Morphological observation of MEL samples using polarized light microscopy. (I) MEL/PVP, (II) MEL/HPC, (III) MEL/EPO, and (IV) crystalline MEL 
the same conditions (Fig. 2b-I, II, III). Of the three ASD formulations of MEL, MEL/EPO was the most physicochemically stable under both of the tested conditions.

\section{Interaction Between MEL and Polymer in ASD Formulations}

On the basis of the recorded ${ }^{1} \mathrm{H}$ NMR spectra (Fig. 4), most of the proton signals of MEL with Eudragit EPO were shifted up-field relative to MEL alone, although no chemical shift drifting was observed in aromatic proton peaks of $\mathrm{H}-7 \quad(\delta=8.02 \mathrm{ppm})$ and $\mathrm{H}-6^{\prime} \quad(\delta=$ $2.32 \mathrm{ppm})$. In contrast, chemical shifts were not observed with the addition of HPC-SSL or PVP K-30. FTIR analyses were conducted for the evaluation of possible molecular interactions between MEL and polymers in the ASD formulations. As shown in Fig. 5a, amide II, sulphone, and thiazole peaks corresponding to crystalline MEL were observed at 1350,1530 , and $1550 \mathrm{~cm}^{-1}$, respectively. There was no significant difference in the amide II, sulphone, and thiazole peaks of MEL in physical mixtures of MEL with Eudragit EPO, HPC-SSL, and PVP K-30. The amide II, sulphone, and thiazole bands of MEL were not transited significantly in MEL/PVP and MEL/HPC. In contrast, significant transitions of the amide II, sulphone, and thiazole bands were observed in the MEL/EPO. For further elucidation, FTIR analyses of MEL/EPO were carried out at various drug concentrations (25\%, 50\%, and $67 \%$ ), as shown in Fig. 5b. Complete transition of the amide II, sulphone, and thiazole bands was observed in $\mathrm{MEL} / \mathrm{EPO}$ at $25 \%$.

\section{Dissolution Behavior of ASD Formulations}

The drug dissolution profiles of crystalline MEL and ASD formulations of MEL at a drug concentration of $25 \%$ were examined in $\mathrm{HCl}$ solution ( $\mathrm{pH}$ 1.2) up to $120 \mathrm{~min}$ (Fig. 6). Crystalline MEL dissolved slowly in acidic medium: after $30 \mathrm{~min}$, only $0.027 \pm 0.002 \%$ of MEL was dissolved in acidic medium. The physical mixtures of MEL with the respective polymers also exhibited limited dissolution behavior (data not shown) like crystalline MEL. In contrast, all ASD formulations showed improved dissolution behaviors in acidic medium compared to that of crystalline MEL. The percentages of dissolved MEL from MEL/PVP, MEL/HPC, and $\mathrm{MEL} / \mathrm{EPO}$ at $30 \mathrm{~min}$ were $3.04 \pm 1.12 \%, 8.65 \pm 1.35 \%$, and $78.1 \pm 6.56 \%$, respectively. The drug dissolution profile of $\mathrm{MEL} / \mathrm{EPO}$ after storage at $40^{\circ} \mathrm{C} / 75 \% \mathrm{RH}$ for 30 days was examined in $\mathrm{HCl}$ solution ( $\mathrm{pH} 1.2$ ) up to $120 \mathrm{~min}$ for confirmation of physicochemical stability. The $f 2$ value of MEL/ EPO before and after storage at $40^{\circ} \mathrm{C} / 75 \% \mathrm{RH}$ for 30 days

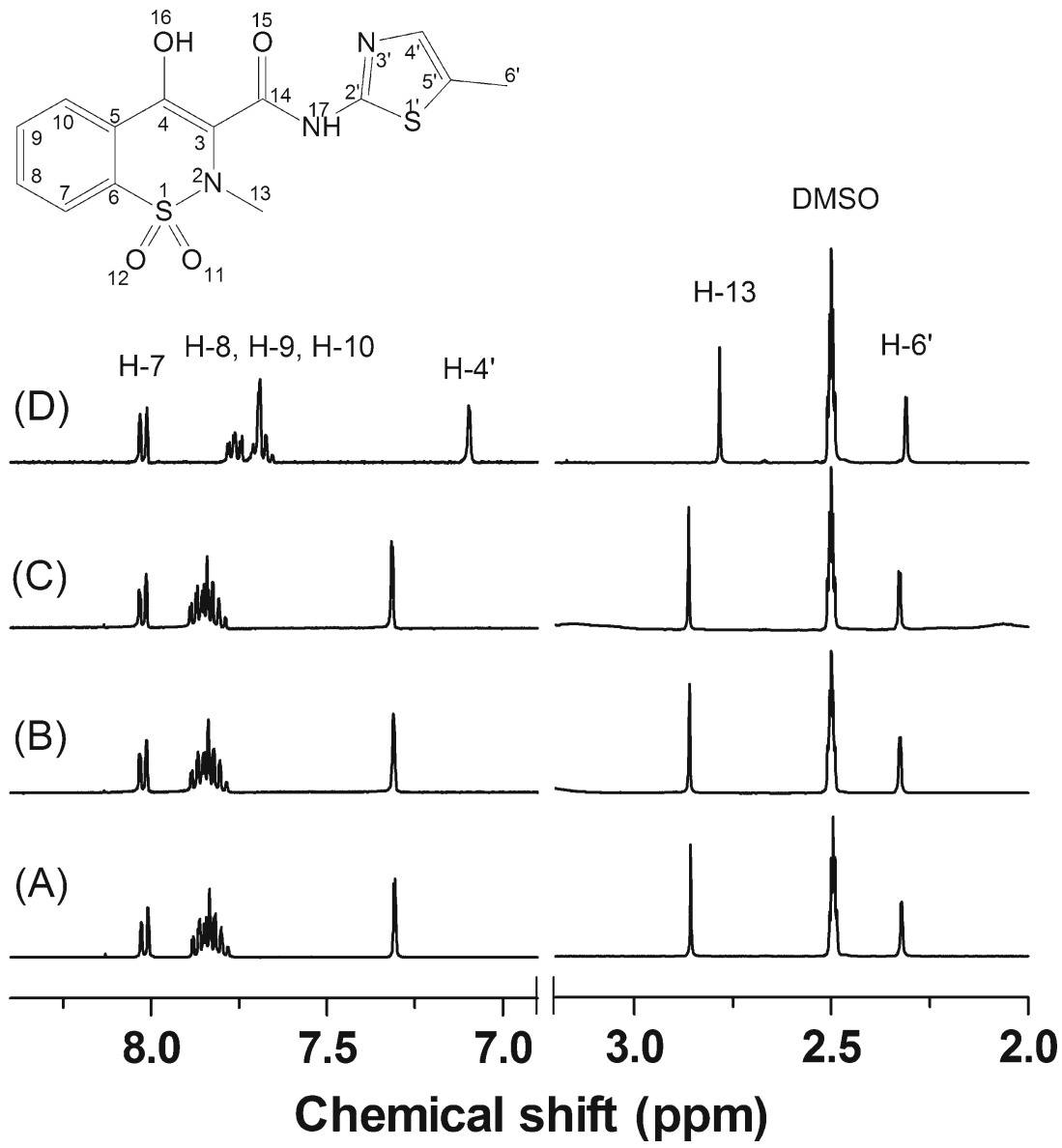

Fig. 4. ${ }^{1} \mathrm{H}$ NMR spectra of MEL with or without Eudragit EPO in $d_{6}$-DMSO. Full spectrum of $(A)$ MEL, $(B)$ MEL with HPC-SSL, $(C)$ MEL with PVP K-30, and $(D)$ MEL with Eudragit EPO. Supramolecular chains of MEL in crystalline form I sustained by sulfonyl amide dimers and sulfathiazole-alcohol supramolecular synthons (15) 
(A)

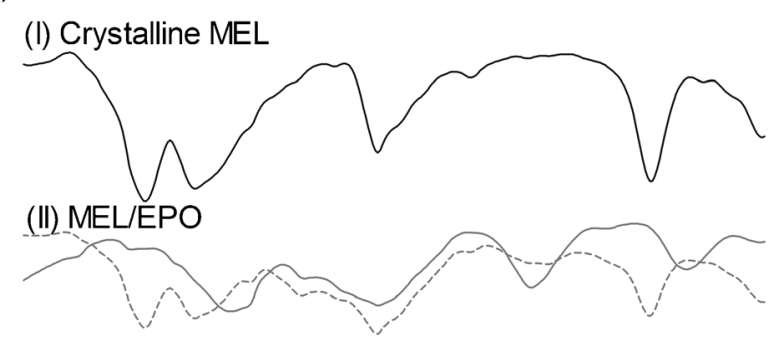

(III) MEL/HPC

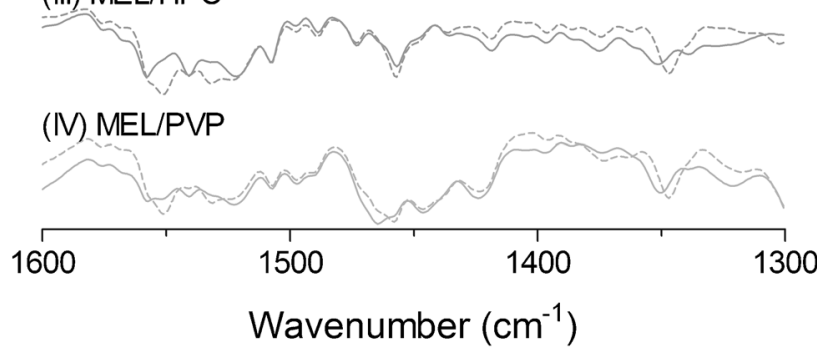

(B)

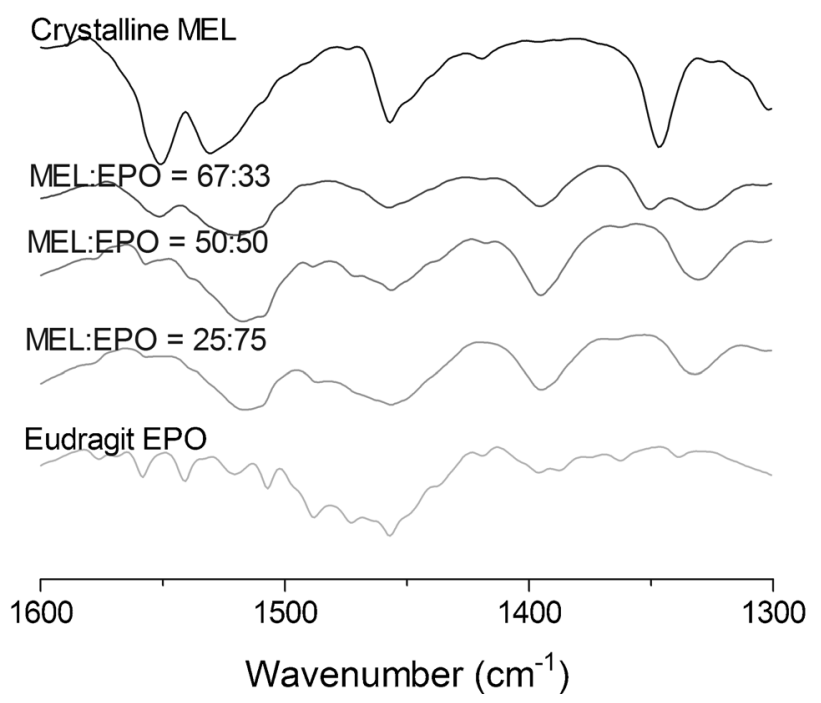

Fig. 5. FTIR spectra of MEL samples. a Baseline-corrected and normalized peak of amide II, sulphone, and thiazole in $(I)$ crystalline MEL, (II) MEL/EPO, (III) MEL/HPC, and (IV) MEL/PVP. b Baseline-corrected and normalized peaks of amide II, sulphone, and thiazole in (I) crystalline MEL, (II) MEL/EPO (67:33), (III) MEL/ EPO (50:50), (IV) MEL/EPO (25:75), and ( $V$ ) Eudragit EPO. Solid line, ASD formulation; broken line, physical mixture of MEL and hydrophilic polymers

was calculated to be 87.97 . Therefore, dissolution profiles were equivalent between before and after storage in MEL/ EPO. However, we could not conduct dissolution testing of $\mathrm{MEL} / \mathrm{PVP}$ and MEL/HPC after storage at $40^{\circ} \mathrm{C} 75 \% \mathrm{RH}$ for 30 days because MEL/PVP and MEL/HPC were converted to semisolid materials.

\section{Pharmacokinetic Profile of a MEL/EPO ASD}

The plasma concentration-time profile of MEL in rats after oral administration of MEL/EPO and crystalline MEL $(1.0 \mathrm{mg}-\mathrm{MEL} / \mathrm{kg})$ is shown in Fig. 7 ; relevant pharmacokinetic

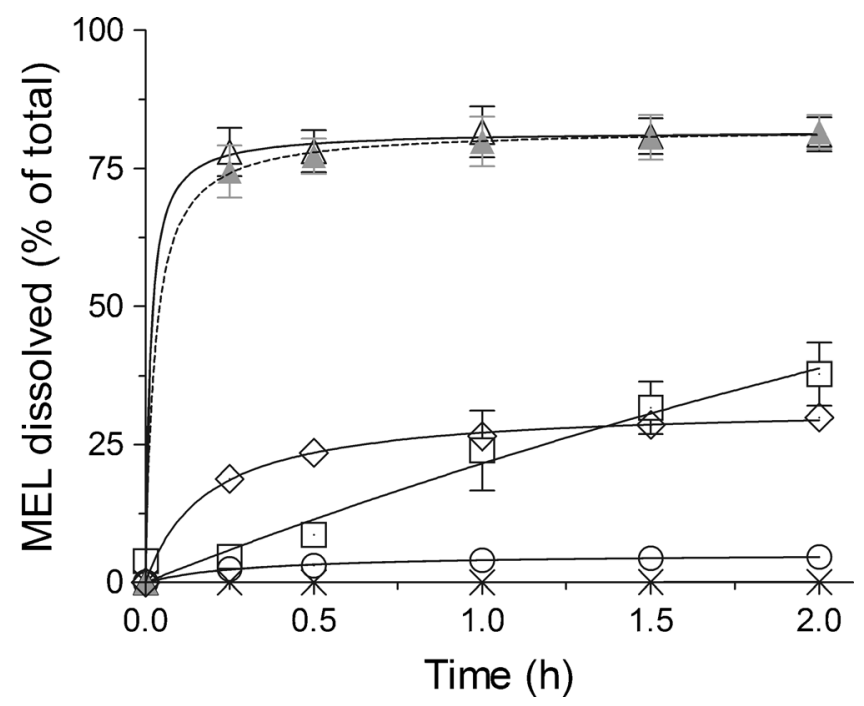

Fig. 6. Dissolution profiles of MEL samples in $\mathrm{pH} 1.2$ medium. MEL/ PVP (O), MEL/HPC ( $\square$ ), MEL/EPO ( $\triangle$ ), MEL/EPO (ム) after storage at $40^{\circ} \mathrm{C} / 75 \% \mathrm{RH}$ for 30 days, marketed formulation $(\diamond)$, and crystalline MEL $(\times)$. Each bar represents the mean \pm SE of three independent experiments

parameters including $C_{\max }$ and $\mathrm{AUC}_{0-8}$ are shown in Table I. Oral administration of crystalline MEL to rats resulted in a gradual elevation in the plasma MEL concentration achieving a $C_{\max }$ of $0.43 \pm 0.10 \mu \mathrm{g} / \mathrm{mL}$. In contrast, the pharmacokinetic behavior of MEL/EPO was better than that in crystalline MEL. Oral administration of MEL/EPO resulted in rapid elevations of plasma MEL levels up to $C_{\max }$ of $1.06 \pm 0.00 \mu \mathrm{g} /$ $\mathrm{mL}$. Thus, $C_{\max }$ and $\mathrm{AUC}_{0-8}$ of MEL/EPO were 2.48- and 2.33-fold, respectively, than that of crystalline MEL.

\section{DISCUSSION}

There have been some studies which develop amorphous solid dispersion of MEL with enhanced dissolution behavior $(9,10)$. However, drug-polymer amorphous solid dispersions

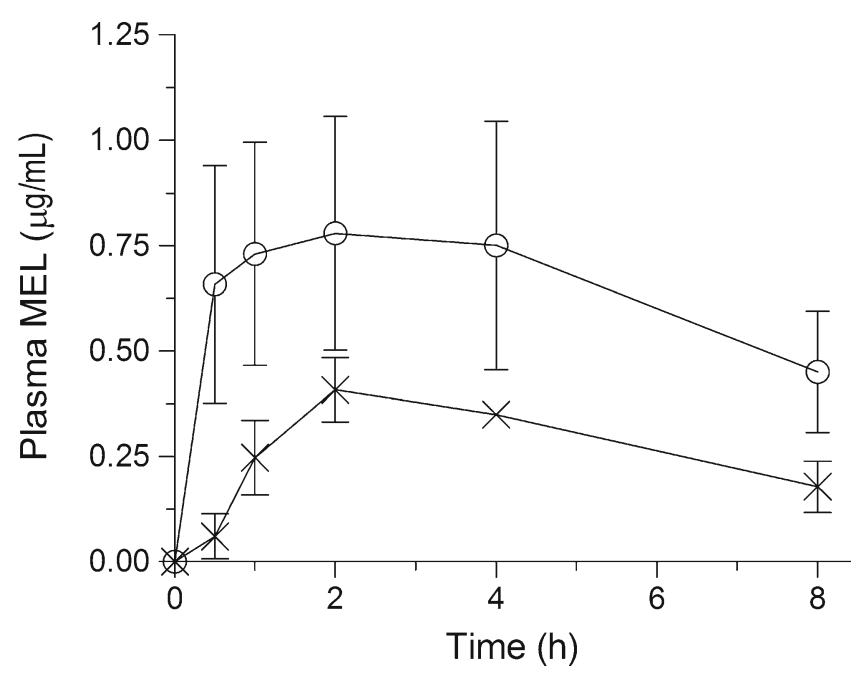

Fig. 7. Plasma MEL concentrations in rats after oral administration of MEL formulation. Crystalline MEL ( $\times$ ), MEL/EPO (O) (1 mg-MEL/kg). Data represent the mean \pm SE of three experiments 
Table I. Pharmacokinetic Parameters of MEL/EPO Following Oral Administration in Rats

\begin{tabular}{lccl}
\hline & Crystalline MEL & MEL/EPO & \\
\hline$C_{\max }(\mu \mathrm{g} / \mathrm{mL})$ & $0.43 \pm 0.10$ & $1.06 \pm 0.00$ & $* * *$ \\
$\operatorname{AUC}_{0-8}(\mu \mathrm{g} \mathrm{h} / \mathrm{mL})$ & $2.21 \pm 0.50$ & $5.20 \pm 1.37$ & $*$ \\
\hline
\end{tabular}

Data represent mean $\pm \mathrm{SD}(n=3)$

$C_{\max }$ maximum concentration, $A U C$ area under the curve of plasma MEL concentration $v s$. time from 0 to $t$ h after oral administration $* P<0.05$ with respect to control (crystalline MEL)

$* * * P<0.001$ with respect to control (crystalline MEL)

of MEL with storage stability have never been reported, although a major issue for ASD is the crystallization of amorphous drugs during long-term storage. Previously, Noolkar et al. developed the stable amorphous solid dispersions of MEL with moringa coagulant and PVP (16). Molecular interaction between MEL and arginine residue of cationic proteins in moringa coagulant is the possible mechanism for stabilization (17). However, moringa coagulant is a heterogeneous mixture of cationic compounds with molecular weight 6$12 \mathrm{kDa}$ and isoelectric point between 10 and 11, obtained by aqueous extraction of Moringa seeds belonging to family Moringaceae. Thus, development of ASD formulation of MEL with clinically used inactive ingredients can contribute for further understandings of the stabilization of MEL ASD and increasing the clinical developability of MEL ASD.

In this study, we obtained three ASD formulations of MEL with PVP K-30, HPC-SSL, and Eudragit EPO. According to Luger et al. and Coppi et al., MEL presents polymorphism with five polymorphic forms, I (enol form), II (zwitterions form), III, IV, and V $(13,14)$; all of the prepared ASDs of MEL showed a halo diffraction pattern; and typical peaks of form I or other known crystalline forms were negligible. Of the three ASDs of MEL, MEL/EPO was the most physicochemically stable under both of the tested conditions. In contrast, typical peaks of crystalline MEL form I were observed by XRPD analysis of MEL/PVP and MEL/HPC after storage at $40^{\circ} \mathrm{C} / 50 \% \mathrm{RH}$ for 30 days (Fig. 2b-I, II) (14).

Intermolecular interaction of drug molecules is necessary for the formation of crystalline. Therefore, the interaction between polymer and drug molecules and interruption of the formation of supramolecular interaction between drug molecules are important factors for decreasing crystallization rate of amorphous drugs (15). ${ }^{1} \mathrm{H}$ NMR analysis revealed the molecular interaction between MEL and Eudragit EPO (Fig. 4). The chemical shift drifting was clearly observed with the addition of Eudragit EPO. In contrast, the addition of HPC-SSL or PVP K-30 did not show significant chemical shit drifting of MEL, suggesting that MEL did not interact with HPC-SSL and PVP K-30. FTIR analyses were conducted for further characterization of the molecular interaction in MEL ASDs (Fig. 5). The amide II, sulphone, and thiazole bands of MEL were not transited significantly in MEL/PVP and MEL/ HPC. In contrast, significant transitions of the amide II, sulphone, and thiazole bands were observed in the MEL/ EPO. MEL molecules form chains sustained by sulfonyl amide dimers and sulfathiazole-alcohol supramolecular synthons in polymorphic form I of crystalline MEL. Therefore, the hydrogen bonding interaction of hydroxyl moiety of MEL with dimethylaminoethyl moiety of Eudragit EPO and interruption of the formation of sulfathiazole-alcohol supramolecular interaction might lead to the inhibition of crystal growth of MEL. These observations were consistent with the previous report, which showed that hydrogen bonding between indomethacin and polymer inhibited the formation of the carboxylic acid dimer that was required for nucleation and growth of the crystal form of indomethacin (15). For further characterization, FTIR analyses of MEL/EPO were carried out at various drug concentrations $(25 \%, 50 \%$, and $67 \%)$, as shown in Fig. 5b. Complete transition of the amide II, sulphone, and thiazole bands was observed in MEL/EPO at $25 \%$. In contrast, the transition of the amide II peak of MEL/EPO at a drug concentration of $67 \%$ was not complete, and the wavelength of the peak was equivalent to that observed with crystalline MEL, indicating slight recrystallization of MEL. Preliminary XRPD experiments also demonstrated that partial recrystallization occurred in MEL/HPC and MEL/PVP at drug concentrations of $50 \%$ or higher (data not shown).

All ASD formulations showed improved dissolution behaviors in acidic medium compared to that of crystalline MEL (Fig. 6). Generally, the amorphous form theoretically represents the most energetic solid-state of the material and thus is expected to yield the largest improvement in dissolution profiles. The enthalpy, entropy, and free energy of an amorphous drug can be much higher than those of the crystalline counterpart (8) so that the excess free energy of an amorphous MEL may result in an improvement in the dissolution behavior. Of all the MEL solid dispersions prepared, MEL/EPO showed the largest improvement in dissolution behavior. Eudragit EPO is a cationic copolymer that dissolves in solution of $\mathrm{pH}<5$; therefore, MEL/EPO is expected to dissolve faster in gastric fluid than MEL/PVP and MEL/ HPC. The typical mechanisms for the improvement of dissolution characteristics of drugs by the ASD approach are the absence of crystallinity and improved wettability (6). Thus, the difference in the crystallinity and wettability of hydrophilic polymers might contribute to make a difference in the dissolution profiles of ASDs. Our observations were consistent with the previous report, which showed that the ASD formulation of poorly soluble tranilast and Eudragit EPO led to a marked improvement in the dissolution properties (5). Taking into account both dissolution properties and physicochemical stability, MEL/EPO was the most reliable ASD formulation for the enhancement of bioavailability among the ASD formulations tested. In previous studies several ASDs of MEL have been reported $(9,10)$. Similar to our outcomes, ASD of MEL with PEG 6000 and PVP K-30 exhibited a significant improvement in dissolution profiles and enhanced in vivo oral absorption. However, storage stabilities of these formulations were not confirmed. Since storage stability is the most concerning issue for ASDs and is indispensable for ensuring therapeutic efficacy, the MEL/EPO formulation has reliable therapeutic potential. Although ulcer was not observed in gastrointestinal tract after single oral administration of MEL/ EPO and crystalline MEL in rats $(1.0 \mathrm{mg}-\mathrm{MEL} / \mathrm{kg}$ body weight), long-term study was not conducted. Daily use of $\mathrm{MEL} / \mathrm{EPO}$ might have higher risk of ulcer in gastrointestinal tract than the marketed formulation because MEL/EPO 
would enhance the dissolved amount of MEL in gastric fluid, leading to increased exposure of MEL to gastric cells. However, patients with rheumatoid arthritis and osteoarthritis often experience acute exacerbations of rheumatism and osteoarthritis; therefore, rapid absorption is important for the relief of pain as early as possible in patients with exacerbations of rheumatism and osteoarthritis. Thus, MEL/EPO would be useful for the temporary treatment in patients with exacerbations of rheumatism and osteoarthritis.

$\mathrm{MEL} / \mathrm{EPO}$ with the marked increase in the dissolution rate and solubility was studied in rats to verify the pharmacokinetic behavior and enhanced bioavailability of this formulation (Fig. 7). The pharmacokinetic behavior of MEL/EPO was improved compared to that of crystalline MEL, yielding increases of 2.48- and 2.33-fold in $C_{\max }$ and $\mathrm{AUC}_{0-8}$, respectively. Our findings suggest the therapeutic potential of MEL/EPO in the treatment of pain. Patients with rheumatoid arthritis and osteoarthritis often experience acute exacerbations of rheumatism and osteoarthritis; therefore, rapid onset of action is important for the relief of pain as early as possible. The mechanism of pharmacological action of MEL, like that of other NSAIDs, is prostaglandin cyclooxygenase inhibition. Ibuprofen sodium dihydrate, a fast-dissolving salt of ibuprofen, has been shown to reduce median time to substantial pain relief by $14 \mathrm{~min}$ in subjects with moderate-to-severe pain after extraction of third molars (18). Thus, MEL/EPO is expected to provide rapid onset of action because of its fast dissolution and rapid absorption.

\section{CONCLUSIONS}

In the present study, three ASD formulations of MEL were prepared and their physicochemical properties were characterized. There were significant improvements in the dissolution behavior of all tested ASD formulations of MEL in acidic solutions; in particular, MEL/EPO exhibited significant improvement in dissolution behavior at $\mathrm{pH}$ 1.2. There was no significant crystallization in MEL/EPO after storage at $40^{\circ} \mathrm{C} / 75 \% \mathrm{RH}$ for 30 days. Systemic exposure of MEL early after oral administration occurred more rapidly with MEL/ EPO than with crystalline MEL. Based on these findings, $\mathrm{MEL} / \mathrm{EPO}$ is expected to be a promising formulation for achieving the rapid pharmacological action of MEL in patients with severe pain.

\section{REFERENCES}

1. Furst DE. Meloxicam: selective COX-2 inhibition in clinical practice. Semin Arthritis Rheum. 1997;26(6):21-7.
2. Hosie J, Distel M, Bluhmki E. Meloxicam in osteoarthritis: a 6month, double-blind comparison with diclofenac sodium. Br J Rheumatol. 1996;35:39-43.

3. Aoki T, Yamaguchi H, Naito H, Shiiki K, Izawa K, Ota Y, et al. Premedication with cyclooxygenase-2 inhibitor meloxicam reduced postoperative pain in patients after oral surgery. Int J Oral Maxillofac Surg. 2006;35(7):613-7.

4. Ghorab MM, Abdel-Salam HM, El-Sayad MA, Mekhel MM. Tablet formulation containing meloxicam and beta-cyclodextrin: mechanical characterization and bioavailability evaluation. AAPS Pharmscitech. 2004;5(4), e59.

5. Kawabata Y, Wada K, Nakatani M, Yamada S, Onoue S. Formulation design for poorly water-soluble drugs based on biopharmaceutics classification system: basic approaches and practical applications. Int J Pharm. 2011;420(1):1-10.

6. Leuner C, Dressman J. Improving drug solubility for oral delivery using solid dispersions. Eur J Pharm Biopharm. 2000;50(1):47-60.

7. Kawakami K. Current status of amorphous formulation and other special dosage forms as formulations for early clinical phases. J Pharm Sci. 2009;98(9):2875-85.

8. Friesen DT, Shanker R, Crew M, Smithey DT, Curatolo WJ, Nightingale JA. Hydroxypropyl methylcellulose acetate succinate-based spray-dried dispersions: an overview. Mol Pharm. 2008;5(6):1003-19.

9. El-Badry M, Fathy M. Enhancement of the dissolution and permeation rates of meloxicam by formation of its freeze-dried solid dispersions in polyvinylpyrrolidone K-30. Drug Dev Ind Pharm. 2006;32(2):141-50.

10. Vijaya Kumar SG, Mishra DN. Preparation, characterization and in vitro dissolution studies of solid dispersion of meloxicam with PEG 6000. Yakugaku Zasshi. 2006;126(8):657-64.

11. Gupta P, Thilagavathi R, Chakraborti AK, Bansal AK. Role of molecular interaction in stability of celecoxib-PVP amorphous systems. Mol Pharm. 2005;2(5):384-91.

12. Miyazaki T, Aso Y, Yoshioka S, Kawanishi T. Differences in crystallization rate of nitrendipine enantiomers in amorphous solid dispersions with HPMC and HPMCP. Int J Pharm. 2011;407:111-8.

13. Luger P, Daneck K, Engel W, Trummlitz G, Wagner K. Structure and physicochemical properties of meloxicam, a new NSAID. Eur J Pharm Sci. 1996;4(3):175-87.

14. Coppi L, Sanmarti MB, Clavo MC, inventors; Crystalline forms of meloxicam and processes for their preparation and interconversion patent US Patent 6,967,248 B2. 2005.

15. Matsumoto T, Zografi G. Physical properties of solid molecular dispersions of indomethacin with poly(vinylpyrrolidone) and poly(vinylpyrrolidone-co-vinyl-acetate) in relation to indomethacin crystallization. Pharm Res. 1999;16(11):1722-8.

16. Noolkar SB, Jadhav NR, Bhende SA, Killedar SG. Solid-state characterization and dissolution properties of meloxicammoringa coagulant-PVP ternary solid dispersions. AAPS PharmSciTech. 2013;14(2):569-77.

17. Bhende S, Jadhav N. Moringa coagulant as a stabilizer for amorphous solids: part I. AAPS PharmSciTech. 2012;13(2):400-10.

18. Schleier P, Prochnau A, Schmidt-Westhausen AM, Peters H, Becker J, Latz T, et al. Ibuprofen sodium dihydrate, an ibuprofen formulation with improved absorption characteristics, provides faster and greater pain relief than ibuprofen acid. Int J Clin Pharmacol Ther. 2007;45(2):89-97. 